\title{
A Directionally Adaptive Edge Anti-Aliasing Filter
}

Konstantine Iourcha*

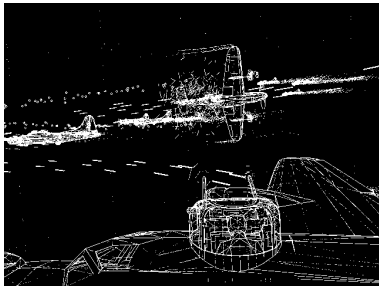

Jason C. Yang ${ }^{\dagger}$

Advanced Micro Devices, Inc.
Andrew Pomianowski ${ }^{\ddagger}$

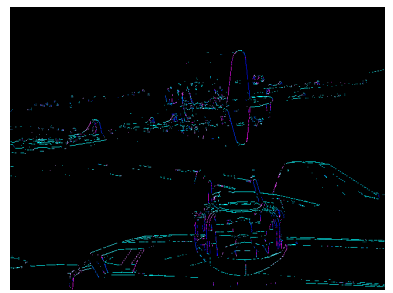

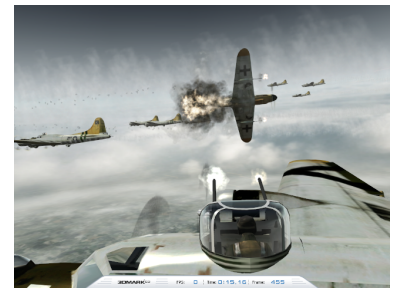

Figure 1: Steps of the Directionally Adaptive Edge Anti-Aliasing Filter algorithm. The left frame shows geometry edge pixels in the scene determined from the hardware MSAA samples. The center frame represents the gradients at the pixels to be filtered. The right frame is the final image where filtered colors for the pixels in the center image are derived using MSAA samples from a neighborhood of 3x3 pixels and 72 subsample values. (Images generated from Futuremark 3DMark03.)

\begin{abstract}
The latest generation of graphics hardware provides direct access to multisample anti-aliasing (MSAA) rendering data. By taking advantage of these existing pixel subsample values, an intelligent reconstruction filter can be computed using programmable GPU shader units. This paper describes an adaptive anti-aliasing (AA) filter for real-time rendering on the GPU. Improved quality is achieved by using information from neighboring pixel samples to compute both an approximation of the gradient of primitive edges and the final pixel color.
\end{abstract}

CR Categories: I.3.3 [Computer Graphics]: Picture/Image Generation-Display Algorithms;

Keywords: anti-aliasing, frame buffer algorithms

\section{Introduction}

As the power and flexibility of graphics hardware increases, more fixed-function operations will be implemented on programmable units. It has already been shown that many post-rendering effects can be implemented on these shader units (e.g., motion blur). A logical step forward is programable anti-aliasing (AA) functionally. By using a shader-based filter, future AA modes could be updated easily with a simple driver change or implemented directly by developers.

We developed an improved post-processing filter that can be implemented for real-time rendering using current GPU capabilities while providing superior edge AA. High-quality results are obtained without significantly increasing the number of hardware samples per pixel, storage resources, or rendering time. As the

*Konstantine.Iourcha@ amd.com
$\dagger$ † JasonC.Yang@amd.com

‡Andrew.Pomianowski@amd.com
GPU's ALU power keeps increasing, it not only becomes feasible to implement fairly complex post-processing, but such a system also has greater efficiency.

In this paper, we describe an improved, shader-based antialiasing filter that takes advantage of new multisample anti-aliasing (MSAA) hardware features exposed through the Microsoft DirectX 10.1 [Mic 2008] API. These features provide direct access to the MSAA sample buffer and the sample patterns used to generate pixel subsamples. The new filtering method computes a more accurate integration of primitive coverage over a pixel by using subsample information for a pixel and its neighbors. This overcomes the pixel scope limitation of existing hardware AA filters. This filter is the basis for the Edge-Detect Custom Filter AA driver feature on ATI Radeon HD GPUs.

\section{Prior Work}

Many solutions to the aliasing problem for computer graphics have been known for some time. [Catmull 1978] introduced an antialiasing method that is the basis for most solutions today. After all polygons in a scene are rendered, a pixel is colored by the contribution of the visible polygons weighted by visibility area. This corresponds to convolution with a box filter.

Other AA contributions include the A-buffer [Carpenter 1984][Schilling and Strasser 1993][Wittenbrink 2001], stochastic sampling [Dippé and Wold 1985][Keller and Heidrich 2001][Akenine-Möller and Ström 2003][Hasselgren et al. 2005][Laine and Aila 2006][Schilling 1991], and multisampling [Akeley 1993][Haeberli and Akeley 1990][Beaudoin and Poulin 2004].

More advanced methods that employ non-box filters include the SAGE graphics architecture, which [Deering and Naegle 2002] uses neighboring pixel information to process up to 400 samples per output. [Sen 2004] stores additional data per pixel to define subpixel edge positions, but this information is generated from manual annotations of processing from computer vision image segmentation techniques. Efficiently implementing this method for our purposes would be difficult.

[Lau Mar 2003] employs lookup tables to filter a pixel. Based on a $5 \times 5$ pixel area, a $1 \mathrm{M}$ entry table is required for a maximum of five different gradations. Unfortunately, these tables would not scale well in our situation as we use up to 72 samples, which would result 

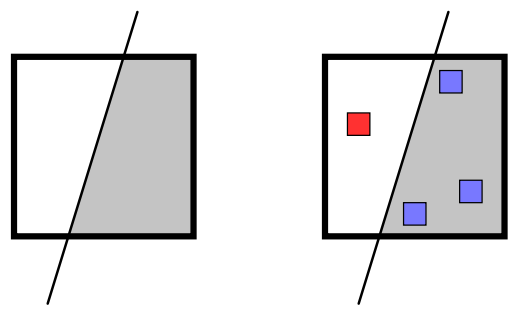

Figure 2: The left pixel shows the area contribution by a primitive. In MSAA, the coverage area is approximated using the sub-pixel samples. On the right, the pixel is considered $3 / 4$ covered.

in a $1 \mathrm{G}+$ entry table. Furthermore, we explicitly try to avoid table usage to avoid consuming GPU memory and bandwidth as well as irregular memory access patterns.

[Rokita 2005] and [Rokita 2006] are extremely simple and inexpensive approaches to AA and would generate too few levels of intensity gradations. Adapting this approach to our requirements would be difficult.

There are relevant works in the adjacent fields of image and video upsampling [Li and Orchard Oct 2001][Zhang and Wu 2006] [Su and Willis 2004][Wang and Ward 2007][Asuni and Giachetti 2008][Giachetti and Asuni 2008][Yu et al. 2001], but most of those algorithms would be difficult to adapt for our purposes. The straightforward application of these algorithms to our problem would be to upscale multisampled images about twice and then integrate samples on the original pixels, but this would require computing 16 to 24 additional samples per-pixel, which has a prohibitively high computational cost. Also, these algorithms are designed around upsampling on Cartesian grids and their adaptation to non-uniform grids (used in hardware multisampling based AA) is not always obvious. Finally, some upsampling methods may not completely avoid edge blurring in the cross direction, which we try to eliminate as much possible.

Our method is closer to those based on isolines such as [Wang and Ward 2007], but we use a much simpler model as no actual upsampling happens (we do not need to calculate new samples; in fact we downsample), nor do we need to process all pixels (we can use a standard resolve for the pixels which do not fit our model well). Moreover, we avoid explicit isoline parameter computation other than the local direction. This allows us to perform the processing in real-time using a small fraction of hardware recourses while still rendering the main application at the same time.

\section{Hardware Anti-Aliasing}

The two most popular approaches to anti-aliasing on the graphics hardware are supersampling and MSAA.

Supersampling is performed by rendering the scene at a higher resolution and then downsampling to the target resolution. Supersampling is expensive in terms of both performance and memory bandwidth. However, the results tend to have high quality, since the entire scene is rendered at a higher resolution. Downsampling is performed by a resolve, which is the aggregation of the samples with filtering.

MSAA is an approximation to supersampling and is the predominant method of anti-aliasing for real-time graphics on GPUs (Figure 2 ). Whenever a pixel is partially covered by a polygon, the single color contribution of the polygon to the pixel at subsample locations

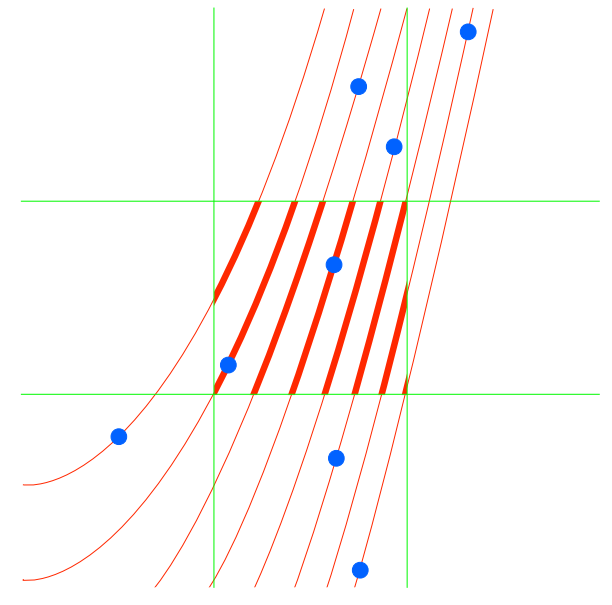

Figure 3: Isolines running through the center pixel with samples used for the function value. Segments inside the pixel are the weights used for integration.

is stored in the MSAA buffer along with the coverage mask [Akeley 1993]. When the scene is ready for display, a resolve is performed. In most implementations, a simple box filter is used that averages the subsample information.

Hardware MSAA modes are characterized by the pattern of the sampling grid. Most graphics hardware employ a non-uniform grid.

We take advantage of the existing hardware by using as input the data stored in the MSAA buffers after rendering. We then replace the standard hardware box-filter with a more intelligent resolve implemented using shaders.

\section{Directionally Adaptive Edge AA Filter}

Our primary goals are to improve anti-alised edge appearance and the pixel coverage estimation when using MSAA on primitive edges with high contrast (Figure 2). In this section we first introduce the filtering method by using a basic single channel example. Then we present the full algorithm details.

\subsection{Single Channel Case}

For simplicity, consider a single channel continuous image (we can use R,G, or B channels or a luma channel of the original image), which can be viewed as a function. To produce an output value for a pixel we need to integrate this function over the pixel area. The standard approximation is to take multiple samples (more or less uniformly distributed) and average them.

If we know isolines of the function, we can use a sample anywhere on the isoline (possibly outside of the pixel area) to determine the function value. Therefore, we can take a set of isoline segments inside the pixel (more or less uniformly distributed) for which we have the sample function values and calculate their weighted average (with the weights being the lengths of the isoline segments inscribed by the pixel) to produce the final pixel value (Figure 3 ). This allows for a more accurate pixel value estimate for the same sample density, as samples outside of the pixel can be used to estimate function values on the isolines, however, we need to calculate the isolines.

If the curvature of the isolines is locally low, we can model them with straight lines. To derive these lines we can compute a tangent 
plane in the center of the pixel and use it as a linear approximation of the function (assuming it is sufficiently smooth). The gradient of this plane is collinear with the gradient of the function and will define the direction of isoline tangents (and approximating straight isolines).

We can extend this model to a discrete world. Having a number of discrete samples (possibly on a non-uniform grid) we can find a linear approximation of the function using a least squares method and use its gradient and isolines as an approximation. Note, if the error of approximation is relatively small, this generally means that the original function is "close to linear" in the neighborhood, the curvature of its isolines can be ignored, and our model works. If, on the other hand, the error is large, this would mean that the model is not valid, and we fall back to a standard sample integration for that pixel (as we generally use a very conservative approach in our algorithm) without creating any artifacts.

The actual images are, however, a three-channel signal, so we need to generalize the above for this case. One way would be to process each channel, but this would considerably increase processing time and may create addition problems when gradients in different channels have vastly different directions. The other possibility, often employed for similar purposes [Yu et al. 2001] is to use only the luminance for isoline determination. However, this would miss edges in chrominance, which is undesirable. Our solution is to follow the framework above and to fit a vector valued linear function of the form described in details below. With that in mind we will still use the terms "gradient approximation" and "isoline approximation" below.

\subsection{Algorithm Overview}

When processing, we are only interested in pixels that are partially covered by primitives. We can determine this by inspecting the MSAA subsamples of a pixel (Section 3). If there are differing subsample color values, we will further process the pixel.

We are not interested in pixels that are fully covered by a primitive (all subsamples having the same values); those pixels are processed as usual (i.e., a box filter). Fully covered (interior) pixels are usually textured and we ignore texture edges because they are pre-filtered or processed by other means.

For pixels that are partially covered, we are mainly interested in those in the middle of long edges (those that extend across several pixels), where jaggies are most visible. Assuming that the isolines and edges do not have high curvature at the pixel, then the three channel signal $f(\boldsymbol{v}) \in \mathbb{R}^{3}$ at the point $\boldsymbol{v}=[x, y]$ can be approximated in the neighborhood of the pixel as

$$
f(\boldsymbol{v}) \approx \tilde{f}(\langle\boldsymbol{g}, \boldsymbol{v}\rangle)
$$

where $\tilde{f}: \mathbb{R}^{1} \rightarrow \mathbb{R}^{3}$ is a function of a scalar argument into color space and $\boldsymbol{g}, \boldsymbol{v} \in \mathbb{R}^{2}$ is the gradient approximation and the point position $[x, y]$ respectively. $\langle$,$\rangle represents a dot product.$

Gradient Calculation We want to find an approximation (1) where $\tilde{f}$ is a linear function which minimizes the squared error:

$$
F=\sum_{i \in I}\left\|\left(C_{1} \cdot\left\langle\boldsymbol{g}, \boldsymbol{v}_{i}\right\rangle+C_{0}\right)-f\left(\boldsymbol{v}_{i}\right)\right\|^{2}
$$

where $I$ is the set of samples in the neighborhood of interest (in our case $3 \times 3$ pixels), $C_{1}, C_{0} \in \mathbb{R}^{3}$ are some constant colors (RGB), and $f\left(\boldsymbol{v}_{i}\right)$ are the color samples. We find an approximation to the

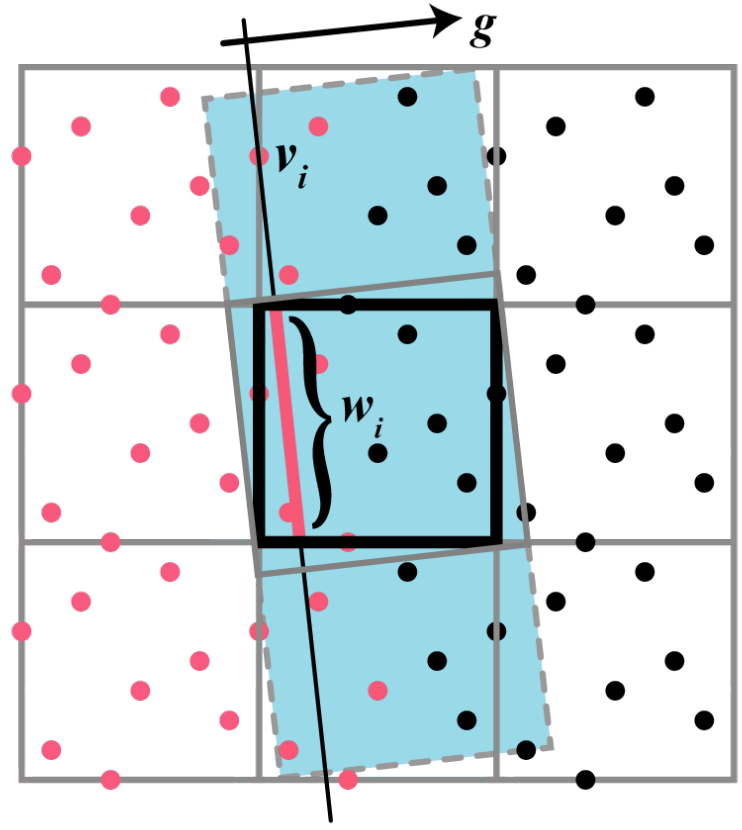

Figure 4: Integration Model. 1) Construct a square around the pixel, with two sides orthoganal to $\boldsymbol{g}(\perp \boldsymbol{g})$. 2) Extend the rectangle, in the direction $\perp \mathbf{g}$ until it meets the $3 \times 3$ pixel boundary. 3) For every sample $\boldsymbol{v}_{i}$, the line segment, from the line passing through the sample and $\perp \mathbf{g}$, enscribed by the pixel is the weight $w_{i}$. 4) Using eq. (5) the pixel color is calculated.

gradient by minimizing $F$ over $C_{0}, C_{1}$, and $g$ using standard least squares techniques [Korn and Korn 1961].

The resulting minimization problem can be solved as follows: First, if $\boldsymbol{v}_{i}$ are centered such that $\sum_{i \in I} \boldsymbol{v}_{i}=0$ (this can be achieved with an approapriate substitution) then $C_{0}$ is the mean of $\left\{f\left(\boldsymbol{v}_{i}\right)\right\}_{i \in I}$, hence we can assume without loss of generality that $\left\{\boldsymbol{v}_{i}\right\}_{i \in I}$ and $\left\{f\left(\boldsymbol{v}_{i}\right)\right\}_{i \in I}$ are both centered. Differentiating on components of $C_{1}$ results in a linear system which can be analytically solved. Substituting this solution for $C_{1}$ into (2) transforms it into a problem of maximizing the ratio of two non-negative quadratic forms. This is essentially $2 \times 2$ eigenvector problems and can be easily solved. Note, that we do not compute $C_{1}$ numerically at all, (as all we need is $g$ ).

If the solution for $\boldsymbol{g}$ is not unique this means that either $C_{1}$ is zero (the function is approximated by a constant) or different image channels (components of $f$ ) do not correlate at all (i.e., there is no common edge direction among the channels). In either case we ignore the pixel. If performance is a concern, the computations can be simplified by using aggregate samples per pixel instead of the original $v_{i}$. For many applications this provides sufficient accuracy. On the other hand, if detection of a particular image feature is needed with higher accuracy, other (possibly non-linear) $\tilde{f}$ can be used, but usually at a much higher computational cost.

Although the accuracy of our integration is dependent on the accuracy of the gradient approximation, we found that errors resulting from error in the gradient estimates are not significant.

Thresholding Of concern are regions with edges of high curvature (i.e., corners) or having non-directional high frequency signal 
where unique solutions of the above least squares problem still exist. Since we assume isolines are locally straight or have low curvature, filtering hard corners with our integration method may cause undesired blurring.

To reduce potential blurring from these cases, we can reject pixels from further processing by using the following thresholding

$$
\begin{gathered}
\delta\left(\boldsymbol{v}_{i}\right)=f\left(\boldsymbol{v}_{i}\right)-\left(C_{1} \cdot\left\langle\boldsymbol{g}, \boldsymbol{v}_{i}\right\rangle+C_{0}\right) \\
\left(\frac{\sum_{i \in I}\left\|\delta\left(\boldsymbol{v}_{i}\right)\right\|^{2}}{\sum_{i \in I}\left\|f\left(\boldsymbol{v}_{i}\right)-C_{0}\right\|^{2}}\right)^{1 / 2} \leq \text { threshold }
\end{gathered}
$$

The pixel passes if eq. (4) holds using a threshold that is relatively small. This would imply that the approximation of eq. (1) is valid. We can also control the amount of blurring by adjusting the threshold.

Note, that if we have an estimate of $\boldsymbol{g}$ in (2), we can use it with $\tilde{f}$ of a different form. So, we could find an optimal step-function $\tilde{f}$ approximation (2) using the obtained $\boldsymbol{g}$ and use it for more precise thresholding. However, the computational cost would be too high for real-time processing and we found that the method provides satisfactory results without it.

Generally, the filtering requirements are application dependent; some professional applications (for instance flight simulators) are required to have a minimal amount of high frequency spacial and temporal noise while bluring is not considered a significant problem. The situation is often opposite in game applications where big amount of high frequency noise can be tolerated (and even at some times this is confused with "sharpness"), but blury images are not appreciated.

Therefore, no "universal threshold" can be specified, but we found that a threshold appropriate for an application can be easily found experimentally; an implementation could have a user adjustable slider.

Stochastic Integration Under assumption (1), the following integration can be used. A gradient-aligned rectangle, which approximately aligns with isolines, is constructed by taking a circumscribed square around the pixel with two sides orthogonal to $g$ and extruding it in the direction orthogonal to $g$ until it meets with the boundary of the $3 \times 3$ pixel area centered at the pixel (Figure 4).

Now consider all the sample positions $\boldsymbol{v}_{i}$ within the resulting rectangle. To calculate the weight $w_{i}$ of a sample $\boldsymbol{v}_{i}$, under the assumption of (1), we take a line passing through the sample orthogonal to $\boldsymbol{g}$ (s.t. $\langle\boldsymbol{g}, \boldsymbol{v}\rangle=\left\langle\boldsymbol{g}, \boldsymbol{v}_{i}\right\rangle$ ). The computed weight $w_{i}$ is equal to the length of the segment of this line enclosed by the pixel. The total result for the pixel is then

$$
\frac{\sum_{i \in I_{R}} f\left(\boldsymbol{v}_{i}\right) \cdot w_{i}}{\sum_{i \in I_{R}} w_{i}}
$$

where $I_{R}$ is the set of indices for the samples inside the rectangle.

Increasing the number of samples, provided they are uniformly distributed, can give a better integral approximation. However, the rectangle cannot be increased too far because the edge in the actual scene might not extend that far out. Visually, in our experiments, the weighting as described works well and provides good performance and quality. Alternatively, the weights could be decreased

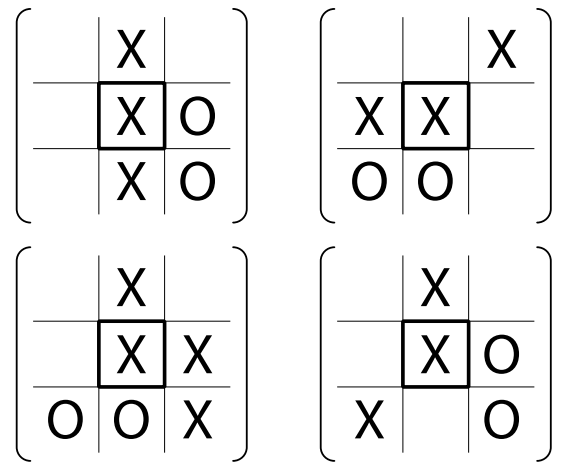

Figure 5: Example pattern masks used to eliminate potential problem pixels. $X$ 's represent edges and $O$ 's represent non-edges. Empty grid spaces can be either edge or non-edge. The edge pixel (at center) would be eliminated from processing if its neighbors do not match one of the patterns.

for samples further from the pixel, but this would reduce the number of color gradations along the edge.

Masking Earlier, we used thresholding from (4) to eliminate potential problem pixels. We can further eliminate pixels by looking at edge patterns within an image. In our implementation, this occurs before finding the gradient.

A $3 \times 3$ grid pattern of edge and non-edge pixels, centered around the candidate pixel, is matched against desired patterns. For example, if only a center pixel is marked as an edge, the pixel is most likely not a part of a long primitive edge and we exclude it from processing. If all pixels in the $3 \times 3$ region are marked as edge pixels, we conservatively assume that no dominating single edge exists and fall-back to standard processing as well. Figure 5 shows a subset of the pattern masks used to classify edges for processing. Defining a complete classifier is a non-obvious task (see discussion in [Lau Mar 2003]).

Any pixels that have been rejected during the entire process (thresholding and masking) are resolved using the standard box filter resolve. In our experiments, we found that pixels evaluated with our method neighboring those of the standard resolve produced consistent color gradients along edges.

\section{Results}

\subsection{Implementation and Performance}

In our implementation, we use four, full-screen shader passes corresponding to each part of the filtering algorithm (see Figure 1 for a subset):

Pass 1 Identify edge pixels using the MSAA buffer. Seed the frame buffer by performing a standard resolve at each pixel.

Pass 2 Mask out candidate pixels using edge patterns.

Pass 3 Compute the edge gradient for pixels that were not rejected in the last pass and use thresholding to further eliminate pixels. Values are written to a floating point buffer.

Pass 4 Derive the final frame buffer color for the pixels from the previous pass through stochastic integration using samples from a $3 \times 3$ pixel neighborhood. Integration and weights are 
calculated in the shader. All other pixels have already been filtered during the first pass.

Shaders were developed using DirectX HLSL Pixel Shader 4.1. All parts of the algorithm are computed in shader with no external tables. Weights and masks are computed dynamically. Pixels that are rejected from subsequent passes can be identified by either writing to a depth buffer or a single channel buffer along with branching in the shader.

We tested the performance of our shader implementation using 8xAA samples on an ATI Radeon HD 4890 running on several scenes from Futuremark 3DMark03. Rendering time for the filter was between 0.25 to $1.7 \mathrm{~ms}$ at $800 \times 600$ resolution, 0.5 to $3 \mathrm{~ms}$ at $1024 \times 768$, and 1 to $5 \mathrm{~ms}$ at $1280 \times 1024$. Each pass refines the number of pixels that need processing, therefore rendering time is dependent on the number of edges in a frame. See Figure 7 for example scenes.

Currently there is some memory overhead due to the multi-pass implementation, but since only a small percentage of pixels in a frame are actually being processed, performance could be improved by using general compute APIs such as OpenCL. Our algorithm should scale with the number of shader processors, so future hardware improvements and features would also improve the rendering time. Also, the number of passes was chosen mostly for performance and could be combined on future hardware.

\subsection{Quality}

Figure 6 compares the results of the new adaptive AA filter against existing hardware AA methods on a near horizontal edge for a scene rendered at $800 \times 600$ resolution. As a comparison, we also rendered the scene at $2048 \times 1536$ with $8 \times$ AA and downsampled to $800 \times 600$ to approximate rendering with supersampling. Our new filter, using existing hardware 4xAA samples, can produce a maximum of 12 levels of gradation. By using existing hardware 8x AA samples, the filter can achieve up to 24 levels of gradation.

Figure 7 compares the new AA filter against the standard box filter over various scenes and at different resolutions. The important characteristics to note in the image are the number of color graduations along the edges and their overall smoothness. Simultaneously it can also be observed that there is no blurring in the direction perpendicular to each edge when compared to methods that use narrow band-pass filters with wider kernels. Effectively, our filter is as good as a standard hardware resolve with 2 to 3 times the number of samples. We also do not observe temporal artifacts with our filter.

The differences between our filter and current hardware AA methods can be perceived as subtle, but our goal was to only improve the quality of edges. These differences are on the same order of magnitude as the differences between 8x and 16x AA or higher. Full screen image enhancement, although a possible future line of work, is outside the scope of this paper.

One drawback with our method is the aliasing of thin objects such as wire, grass blades, etc. There are cases where the given sampling density within a pixel is not capable of reproducing the object, but the object is detected in neighboring pixels and potentially resulting in gaps in the on-screen object rendering. Although it is possible to try to detect and correct these gaps to a point (i.e., through the masking step), the better solution is higher sampling resolution.

\section{Future Work and Conclusions}

There are several avenues for future research. Better but costlier edge detection algorithms could be used to find pixels amenable to processing. This includes other edge patterns for the refinement of potential edge pixels. Instead of using the existing MSAA samples and coverage masks, our algorithm could be applied to supersampling as well as filtering pixels with edges in textures. It might be possible to improve results by designing a better subsample grid. Finally, it might be possible to adapt our method to image upsampling.

We have presented an improved anti-aliasing filter compared to current hardware methods using new hardware features exposed using DirectX 10.1. Our filter is another example of rendering improvements using increased programmability in hardware. Improved anti-aliasing is an obvious use for the new MSAA features and we expect future developers to find more interesting applications.

\section{Acknowledgements}

The authors would like to thank to Jeff Golds for help in the implementation.

\section{References}

AKELEY, K. 1993. Reality engine graphics. In SIGGRAPH '93: Proceedings of the 20th annual conference on Computer graphics and interactive techniques, ACM, New York, NY, USA, 109116.

AKenine-Möller, T., AND Ström, J. 2003. Graphics for the masses: a hardware rasterization architecture for mobile phones. ACM Trans. Graph. 22, 3, 801-808.

Asuni, N., AND GiachetTi, A. 2008. Accuracy improvements and artifacts removal in edge based image interpolation. In VISAPP (1), 58-65.

Beaudoin, P., And Poulin, P. 2004. Compressed multisampling for efficient hardware edge antialiasing. In GI '04: Proceedings of Graphics Interface 2004, Canadian HumanComputer Communications Society, 169-176.

CARPEnTER, L. 1984. The A-buffer, an antialiased hidden surface method. In SIGGRAPH '84: Proceedings of the 11th annual conference on Computer graphics and interactive techniques, ACM Press, New York, NY, USA, 103-108.

CATMUll, E. 1978. A hidden-surface algorithm with anti-aliasing. In SIGGRAPH '78: Proceedings of the 5th annual conference on Computer graphics and interactive techniques, ACM Press, New York, NY, USA, 6-11.

DeERING, M., AND NAEgLE, D. 2002. The sage graphics architecture. In SIGGRAPH '02: Proceedings of the 29th annual conference on Computer graphics and interactive techniques, ACM Press, New York, NY, USA, 683-692.

DipPÉ, M. A. Z., AND Wold, E. H. 1985. Antialiasing through stochastic sampling. In SIGGRAPH '85: Proceedings of the 12th annual conference on Computer graphics and interactive techniques, ACM Press, New York, NY, USA, 69-78.

Giachetti, A., AND Asuni, N. 2008. Fast artifacts-free image interpolation. In British Machine Vision Conference.

HAEBerli, P., AND AKELEy, K. 1990. The accumulation buffer: hardware support for high-quality rendering. In SIGGRAPH '90. Proceedings of the 17th annual conference on Computer graphics and interactive techniques, ACM, New York, NY, USA, 309318. 

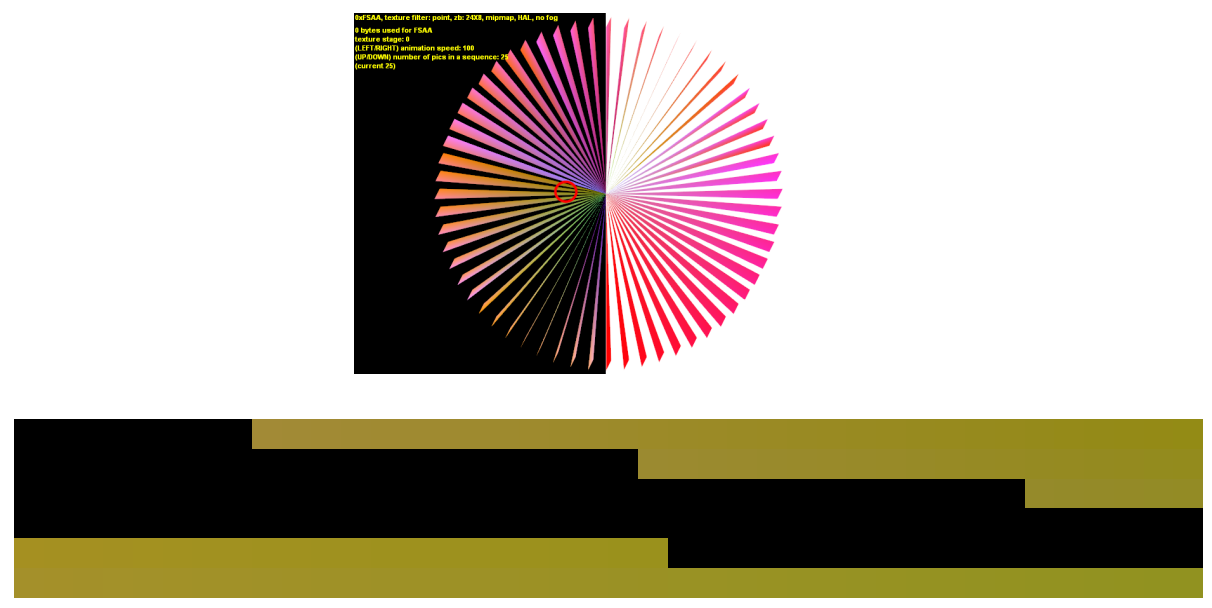

(a) No AA

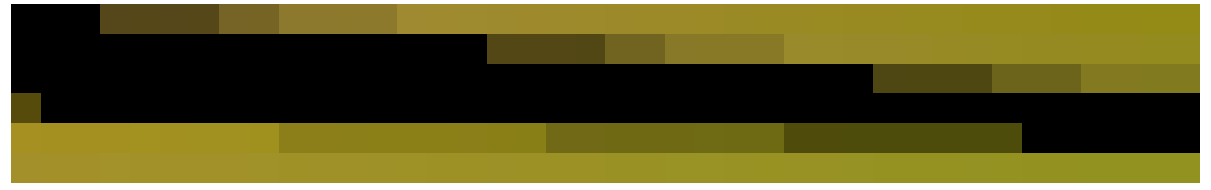

(b) $4 \mathrm{xAA}$

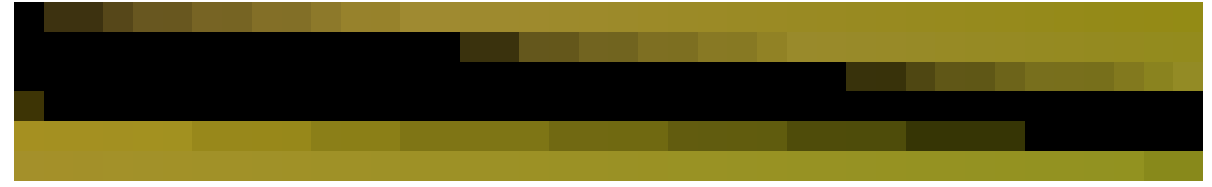

(c) $8 \mathrm{xAA}$

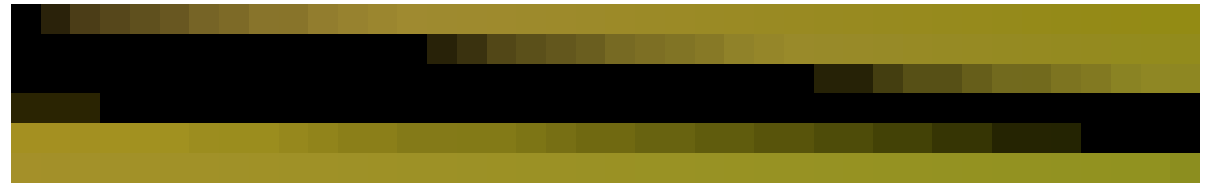

(d) $16 \mathrm{xAA}$

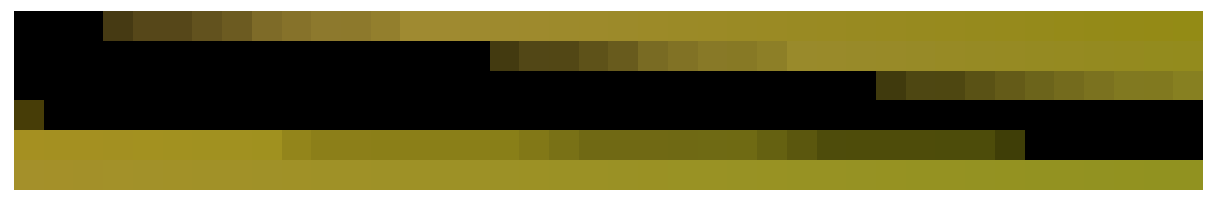

(e) New filter using 4xAA samples

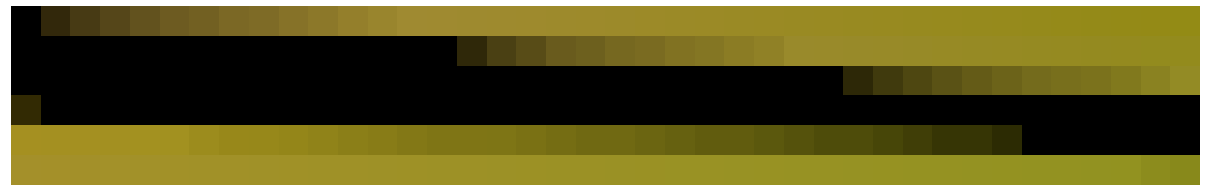

(f) New filter using 8xAA samples

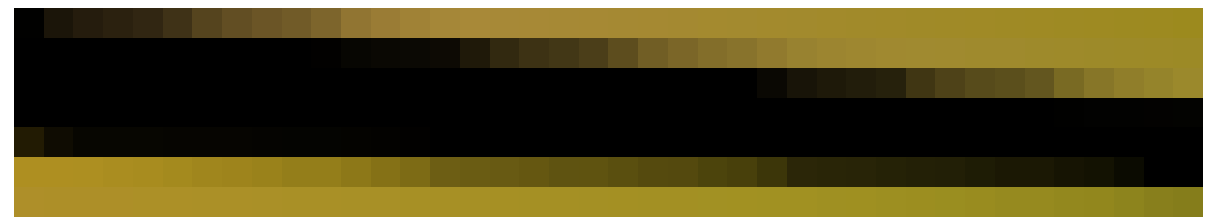

(g) Downsampled from high resolution rendering

Figure 6: A comparison of different AA methods applied to a pinwheel from FSAA Tester by ToMMTi-Systems (top) rendered at 800x600. Shown is a subset of the scene where the edges are at near horizontal angles. (e) shows the new filter using hardware 4xAA samples. In this example, 10 levels of gradation is visually achieved. $(f)$ is the new filter using $8 x A A$ samples. In this example 22 levels of gradation is visually achieved. $(d)$ is Nvidia's 16Q AA filtering. $(g)$ is a downsample of the original scene rendered at $2048 \times 1536$ and $8 \times A A$. 

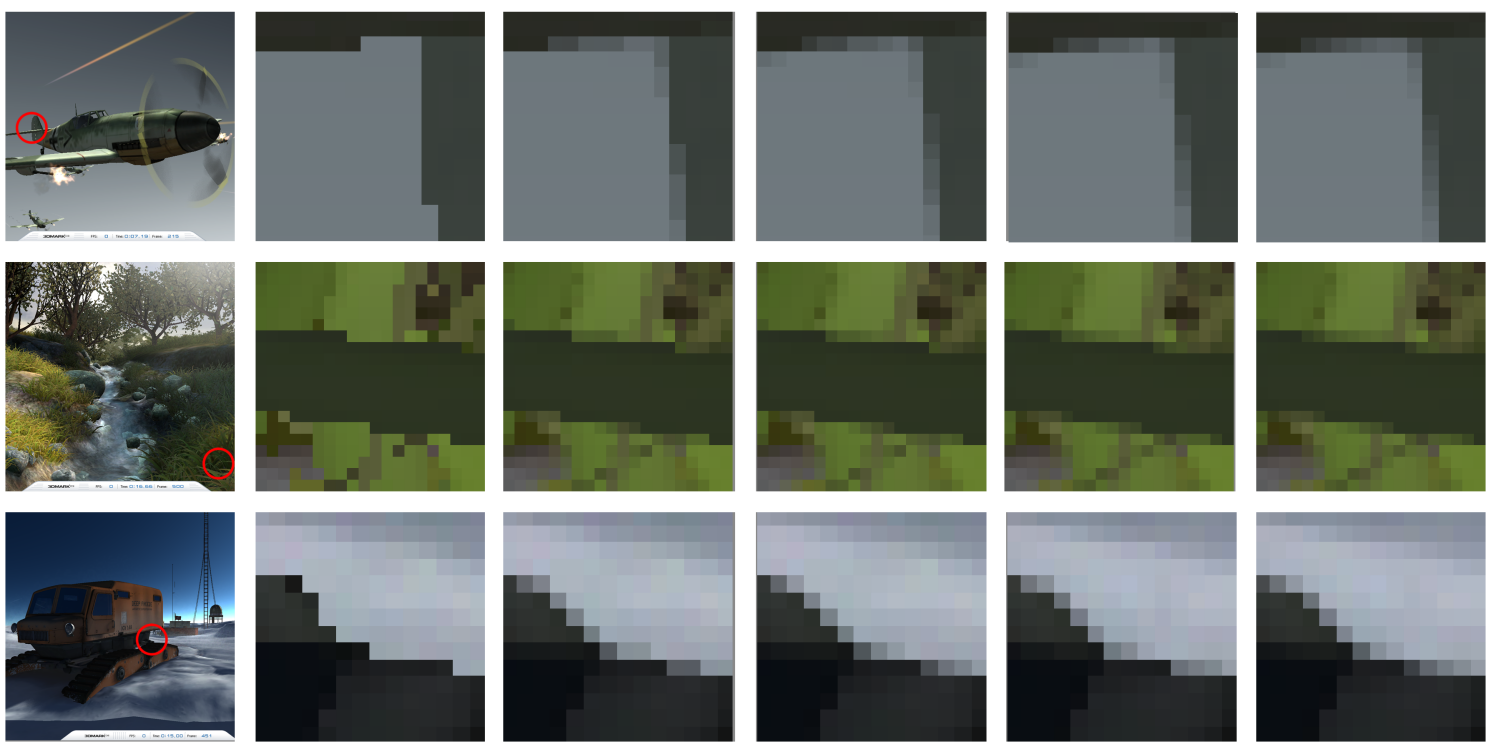

(b) NoAA

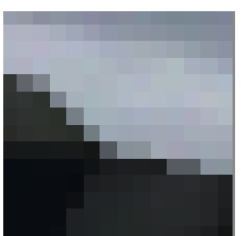

(c) $4 \mathrm{xAA}$

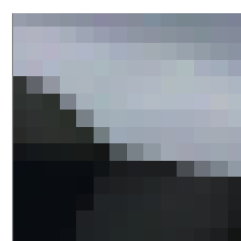

(d) New 4xAA

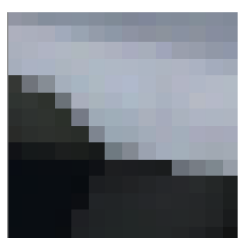

(e) $8 \times A A$

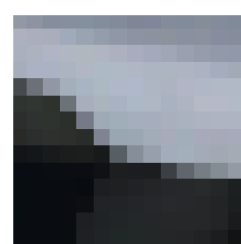

(f) New 8xAA

Figure 7: Comparison of the various AA filters applied on various scenes and at different resolutions. Column (b) is no AA enabled. (c) and $(e)$ are the standard $A A$ resolve at $4 x$ and $8 x$ samples respectively. $(d)$ and $(f)$ are results from the new filtering using the existing $4 x$ and 8x MSAA samples respectively. The first and second rows (Futuremark 3DMark03) are rendered at $800 x 600$ resolution and the third row (Futuremark 3DMark06) is rendered at 1024x768.

Hasselgren, J., Akenine-Möller, T., And Hasselgren, J. 2005. A family of inexpensive sampling schemes. Computer Graphics Forum 24, 4, 843-848.

KELler, A., AND HeIDRICH, W. 2001. Interleaved sampling. In Proceedings of the 12th Eurographics Workshop on Rendering Techniques, Springer-Verlag, London, UK, 269-276.

Korn, G., AND KoRn, T. 1961. Mathematical Handbook for Scientists and Engineers. McGraw-Hill, Inc.

LAIne, S., AND Aila, T. 2006. A weighted error metric and optimization method for antialiasing patterns. Computer Graphics Forum 25, 1, 83-94.

LAU, R. Mar 2003. An efficient low-cost antialiasing method based on adaptive postfiltering. Circuits and Systems for Video Technology, IEEE Transactions on 13, 3, 247-256.

LI, X., AND ORChard, M. Oct 2001. New edge-directed interpolation. IEEE Transactions on Image Processing 10, 10, 15211527.

Microsoft Corporation. 2008. DirectX Software Development Kit, March 2008 ed.

Myszkowski, K., Rokita, P., And TAWARA, T. 2000. Perception-based fast rendering and antialiasing of walkthrough sequences. IEEE Transactions on Visualization and Computer Graphics 6, 4, 360-379.

RoKITA, P. 2005. Depth-based selective antialiasing. Journal of Graphics Tools 10, 3, 19-26.

RoKITA, P. 2006. Real-time antialiasing using adaptive directional filtering. In Real-Time Image Processing 2006. Proceedings of the SPIE., vol. 6063, 83-89.

Schilling, A., AND Strasser, W. 1993. Exact: algorithm and hardware architecture for an improved a-buffer. In SIG-
GRAPH '93: Proceedings of the 20th annual conference on Computer graphics and interactive techniques, ACM, New York, NY, USA, 85-91.

SCHILling, A. 1991. A new simple and efficient antialiasing with subpixel masks. In SIGGRAPH '91: Proceedings of the 18th annual conference on Computer graphics and interactive techniques, ACM Press, New York, NY, USA, 133-141.

SEN, P. 2004. Silhouette maps for improved texture magnification. In HWWS '04: Proceedings of the ACM SIGGRAPH/EUROGRAPHICS conference on Graphics hardware, ACM, New York, NY, USA, 65-73.

Su, D., AND WILLIS, P. 2004. Image interpolation by pixel-level data-dependent triangulation. Computer Graphics Forum 23, 2, 189-201.

WANG, Q., AND WARD, R. 2007. A new orientation-adaptive interpolation method. Image Processing, IEEE Transactions on 16, 4 (April), 889-900.

WITTENBRINK, C. M. 2001. R-buffer: a pointerless a-buffer hardware architecture. In HWWS '01: Proceedings of the ACM SIGGRAPH/EUROGRAPHICS workshop on Graphics hardware, ACM, New York, NY, USA, 73-80.

Yu, X., Morse, B. S., AND Sederberg, T. W. 2001. Image reconstruction using data-dependent triangulation. IEEE Comput. Graph. Appl. 21, 3, 62-68.

ZHANG, L., AND WU, X. 2006. An edge-guided image interpolation algorithm via directional filtering and data fusion. Image Processing, IEEE Transactions on 15, 8 (Aug.), 2226-2238. 